\title{
Breast Cancer in Women Younger Than 35 Years: Features and Outcomes (About 93 Cases)
}

\author{
Meryem Belmajdoub*, Fatima Zehra Fdili Alaoui, Sofia Jayi, Hekmat Chaara, Moulay Abdelilah Melhouf \\ Department of Obstetrics and Gynecology, Hassan II University Hospital in Fez, Morocco
}

\begin{tabular}{ll}
\hline DOI: $\underline{10.36348 / \text { SIJOG.2019.v02i10.003 }}$ & | Received: $16.09 .2019 \mid$ Accepted: $23.09 .2019 \mid$ Published: 30.10 .2019 \\
*Corresponding author: Belmajdoub Meryem &
\end{tabular}

\section{Abstract}

Breast cancer is the first cancer of women in the world. With nearly 1.7 million new cases diagnosed each year around the world, breast cancer is the most common cancer in women and the major public health problem. In Morocco, its incidence continues to increase. Its occurrence is a dramatic event in a woman's life, it is still unhappy and difficult to accept when it is a young woman. This is a retrospective study of 93 patients under 35 years of age with breast cancer who were recruited to the Gynecology and Obstetrics II Department of Hassan II University Hospital in Fez, Morocco, over a period of 5 years. The purpose of the study was an analysis of clinical, therapeutic, evolutionary and prognostic data for breast cancer in women aged 35 and under. The prognosis of breast cancer in our young patients is unfavorable, seems to be related to an advanced stage of the disease, but also a particular biological profile, as well as the data of the literature.

Keywords: Breast cancer, Young woman, Clinico-pathological characteristics, Specific management, prognosis.

Copyright @ 2019: This is an open-access article distributed under the terms of the Creative Commons Attribution license which permits unrestricted use, distribution, and reproduction in any medium for non-commercial use (NonCommercial, or CC-BY-NC) provided the original author and source are credited.

\section{INTRODUCTION}

The definition of young age in women with breast cancer is not unambiguous. Different studies have defined a "young" woman as corresponding to a woman under $30,35,40,45$ years old or simply not premenopausal. In the United States and Europe, the incidence of breast cancer in women under 35 is around $3 \%$ [1]. In Morocco, its incidence continues to increase. Its occurrence is a dramatic event in the life of a woman, it is still poorly felt and difficult to accept when it comes to a young woman. The forms are more aggressive and the diagnosis is more difficult because of the mammary density, the mammography is therefore less efficient. The occurrence of breast cancer at this age may pose psychological problems, but also problems of fertility and sexuality in connection with the aggressive treatments delivered [2]. This retrospective work aims to study the different epidemiological, clinical, pathological, therapeutic and prognostic aspects of breast cancer in young women aged 35 and under.

\section{MATERIAL AND METHOD}

This is a retrospective study spanning a period of 5 years and involving 93 cases of breast cancer in young women aged 35 and under who have been managed by the department of Gynecology and Obstetrics and as well as the department of Oncology of Hassan II University Hospital in Fez. All cases were confirmed histologically, 2forms were bilateral. Methodologically, all the information was centralized on the Access software, which allowed the analysis of the results.

\section{RESULTS \\ Epidemiological and Socio-Economic Characteristics During the period in which the study was conducted, approximately 1275 women were diagnosed with BC, 93 of them were under 35 years of age; which represents, statistically, a frequency of $7.29 \%$. The average age was 31 years with extremes of 17 and 35 years. Median was 30 years oldand whose age group between 31 and 35 years was the most affected. The epidemiological and anatomical clinical characteristics of the patients concerned are well summarized in Table- 1.}


Antecedents

\section{Gynecological Obstetric Antecedents}

The age of menarche was clarified in 22 patients, of whom 14 or $15.05 \%$ had a menarche at an age> 12 years. The parity was specified in 51 patients with a predominance of pauciparity and nulliparity with respective rates of $37.63 \%$ and $11.82 \%$. The age of first pregnancy in patients was 22 years on average with extremes of 16 to 35 years. Contraceptive use was specified in 10 patients of whom 5 or $5.37 \%$ confirmed oral contraceptive use for an average of 8 months. The notion of exclusive breastfeeding was collected in 30 patients or $32.25 \%$ and this for a period of 22 months.

\section{Family history of Breast Cancer}

Seven patients $(7.52 \%)$ had a family history of breast cancer: two in the first degree and five in the second degree.

\section{Clinical Data}

The average time of consultation was 9.17 months witha proportion of $43.01 \%$ of patients consulted beyond 6 months. The reason for consultation was a mammary mass in almost all cases (88.17\%). Pathology most often involved the left breast (55.91\%). Bilateral involvement was observed in 2 patients $(2.15 \%)$. A predominance of $\mathrm{T} 2$ forms was observed in 34 patients, $(36.55 \%)$, followed by $\mathrm{T} 1$ forms in 29 patients $(31.18 \%)$. Ganglionic involvement was noted in 42 patients $(45.16 \%)$, whereas metastasis was present in $17(18.27 \%)$ of patients. The clinical characteristics of the patients are reported in Table-1.
Anatomo-Pathology and Immunohistochemistry

The histological study found $83.87 \%$ of ductal carcinoma infiltrant (CCI). Some rare histological types were also found (Table-2). The immunohistochemical study in 47 patients found positive hormone receptors in 38 patients $(40.86 \%)$ and HER-2 oncoprotein overexpressed in 18 patients $(19.35 \%)$.

\section{Therapeutic Management \\ Chemotherapy}

It was performed in 67 patients $(72.04 \%)$; she was neoadjuvant in 26 patients $(27.95 \%)$ for locally advanced tumors of which 17 patients were metastatic $(18.27 \%)$.

\section{Surgical Treatment}

We performed primary surgery in 67 patients $(72.04 \%)$ and post-chemotherapy surgeryin 11 patients $(11.82 \%)$. It was radical in $40.86 \%$ of them and conservative in 20 patients $(21.50 \%)$.

\section{Other Treatments}

Hormone therapy and radiotherapy were performed in $59.13 \%$ and $72.04 \%$ of patients, respectively. Theanatomo-pathological and therapeutic data are reported in Table- 2 .

\section{Evolution and Prognosis}

The overall survival time was calculated between the date of the diagnosis of breast cancerand last consultation, 42 patients were lost to follow-up, 13 patients died due to their metastatic status (pulmonary, bony, Hepatitis) and 38 patients alive, overall survival at 5 years was $69.96 \%$.

Table-1: Distribution of patients according to epidemiologic and clinical characteristics

\begin{tabular}{|c|c|c|}
\hline Variables & Number Of Cases & Percentage $(\%)$ \\
\hline \multicolumn{3}{|l|}{ age (years) } \\
\hline$<21$ & 2 & $2.15 \%$ \\
\hline 21 à 25 & 2 & $2.15 \%$ \\
\hline 26 à 30 & 12 & $12.90 \%$ \\
\hline 31 à 35 & 77 & $\mathbf{8 2 . 7 9 \%}$ \\
\hline \multicolumn{3}{|l|}{ Family history of breast cancer } \\
\hline Yes & 7 & $7.52 \%$ \\
\hline First degree & 2 & \\
\hline Second degree & 5 & \\
\hline No & 86 & $92.58 \%$ \\
\hline \multicolumn{3}{|l|}{ Consultation time (mouths) } \\
\hline$\leq 1 \mathrm{mois}$ & 12 & $12.90 \%$ \\
\hline 1mois_3mois & 20 & $21.50 \%$ \\
\hline 3mois_6mois & 21 & $22.58 \%$ \\
\hline$>6$ mois & 40 & $43.01 \%$ \\
\hline \multicolumn{3}{|l|}{ the breast reaches } \\
\hline right & 39 & $42 \%$ \\
\hline left & 52 & $55.91 \%$ \\
\hline bilateral & 2 & $2.15 \%$ \\
\hline \multicolumn{3}{|l|}{ Stage $\mathbf{T}$} \\
\hline $\mathrm{T} 1$ & 29 & $31.18 \%$ \\
\hline $\mathrm{T} 2$ & 34 & $36.55 \%$ \\
\hline $\mathrm{T} 3$ & 6 & $6.45 \%$ \\
\hline $\mathrm{T} 4$ & 24 & $25.81 \%$ \\
\hline \multicolumn{3}{|l|}{ Stage N } \\
\hline No & 51 & $54.83 \%$ \\
\hline N1 & 36 & $38.7 \%$ \\
\hline $\mathrm{N} 2$ & 5 & $5.37 \%$ \\
\hline N3 & 1 & $1.07 \%$ \\
\hline \multicolumn{3}{|l|}{ Stage M } \\
\hline M0 & 76 & $81.72 \%$ \\
\hline M1 & 17 & $18.27 \%$ \\
\hline
\end{tabular}


Meryem Belmajdoub; Sch Int J Obstet Gynec, Oct. 2019; 2(10): 252-256

Table-2: Distribution of patients according to histologic and therapeutic characteristics

\begin{tabular}{|l|l|l|}
\hline Variables & Number Of Cases & Percentage (\%) \\
\hline Histological type & & \\
CCI & 81 & $\mathbf{8 7 . 0 9 \%}$ \\
CIS high grade & 6 & $6.45 \%$ \\
phyllode tumor grade 3 & 3 & $3.22 \%$ \\
CCI+CIS & 3 & $3.22 \%$ \\
\hline Expression of hormone receptors (N = 68) & & \\
Positif & 55 & $59.13 \%$ \\
Negative & 13 & $13.97 \%$ \\
\hline Overexpression of oncoprotein HER-2 $(\mathbf{N}=\mathbf{6 8})$ & & \\
Overexpression & 40 & $40 \%$ \\
absence of overexpress & 28 & $30.10 \%$ \\
\hline Chemotherapy & & \\
Neoadjuvant & 26 & $27.95 \%$ \\
Adjuvant & 17 & $18.27 \%$ \\
\hline Surgical treatment & & \\
conservative treatment & 20 & $21.50 \%$ \\
radical treatment & 40 & $40.86 \%$ \\
\hline Radiotherapy & & \\
Yes & 67 & $72.04 \%$ \\
No & 11 & $27.96 \%$ \\
\hline Hormone therapy & & \\
Yes & 55 & $59.13 \%$ \\
No & 38 & $40.87 \%$ \\
\hline
\end{tabular}

\section{DISCUSSION}

Recent literature identifies a group of "very young women" patients with breast cancer before the age of 35 . In terms of epidemiology, about $2 \%$ of breast cancers occur before age 35 in industrialized countries $[1,2]$. The analysis of European registers reveals a moderate $(+0.65 \%$ per year in France), but constant, increase in the incidence of invasive cancers in this group of patients [3]. Young age is recognized as an independent and unfavorable prognostic factor, with a significantly greater risk of recidivism, both locally and remotely. The establishment of a specific pathological profile must be investigated to improve the therapeutic management [4].

\section{Clinico Pathological Characteristics at Diagnosis}

As routine screening by mammography is not intended for these patients, the reason for the consultation is most often the palpation of a nodule or the modification of the appearance of the breast. The clinical size of the tumor at the first consultation is often large, on average $3 \mathrm{~cm}$. There is frequently axillary lymph node involvement. There are tumors of higher histological grade, more aggressive (grade SBR III). The histological type most commonly encountered for infiltrating cancers is the ductal type. The rare medullary tumors $(<3 \%$ of breast cancers) are observed more frequently in these young patients. Multicentric tumors and the presence of lympho-vascular emboli are common. Hormonal receptors are less often positive (68\% in those under 40 years of age compared to $80 \%$ in those aged 40 and over). HER2 is more frequently overexpressed than in postmenopausal women. The tumor proliferation index, measured by KI 67, is higher. The presence of a lymphoid stroma within the tumor is reported and correlated with the risk of local recurrence.
Triple negative tumors are more commonly seen in these young patients. These cancers are associated with a BRCA1 mutation and have a particular sensitivity to certain cytotoxic agents such as platinum salts [5].

\section{Surgical Management}

In these young women, with a tumor often aggressive with a risk of significant local relapse, the choice of mastectomy versus conservative surgery should be discussed in Multidisciplinary Oncology Consultation (COM) and explained to the patient. Neoadjuvant chemotherapy has reduced the number of mastectomies. In conservative surgery, the Milan school has always advocated wide glandular resection which means quadrantectomy. Classic remodeling techniques allow this attitude. Oncoplastic remodeling techniques should be reserved for well-discussed cases. During a conservative surgery, the quality of the excision is related to the integrity of the surgical banks. There are no international recommendations, but findings from meta-analyzes have established a healthy margin threshold at more than $2 \mathrm{~mm}$ from the tumor. These data must always be integrated into the clinicalradiological context. Radiotherapy should always be performed after conservative surgery. Chemotherapy has a significant local protective effect: a local relapse rate of $13 \%$ after chemotherapy versus $28 \%$ without chemotherapy [7]. Body integrity, sexuality, postoperative rehabilitation, the consequences of chemotherapy, radiotherapy, the possibility of a subsequent pregnancy, breastfeeding are factors to discuss with the patient for a "shared decision" [8]. 
Radiation therapy in conservative breast treatment in young women

The recommended treatment is radiotherapy of the mammary gland of $50 \mathrm{GY}$ with a boost of $16 \mathrm{GY}$ on the tumor bed [9]. Intraoperative radiotherapy is not recommended in young patients.

\section{Place of Neoadjuvant Treatments in Young Women}

The main objective of neo-adjuvant treatments is to be able to propose a conservative surgery if the answer is favorable. There is an increase in the rate of tumerectomie of the order of $12 \%$ after chemotherapy. There is no difference in overall survival or survival without metastatic relapse between adjuvant and neoadjuvant chemotherapy. In the case of tumors overexpressing HER2, the neo-adjuvant strategy allows for the early introduction of trastuzumab (Herceptin ${ }^{\circledR}$ ) in combination with chemotherapy [10]. Ithas been shown that the quality of the histological response after neoadjuvant chemotherapy at the level of the mammary tumor and axillary lymph nodes is very important. A complete histological response is considered a powerful prognostic factor for treatment efficacy, with a lower risk of relapse. Tumors with HER2-overexpressing hormone-receptor negative, high histological grade, with a proliferative index (KI 67) high and infiltrating ductal type, are more chemo-sensitive. In neoadjuvant approaches, an optimal locoregional assessment by mammography and ultrasound is recommended, supplemented by MRI if necessary or as part of studies [11]. Patients with extensive microcalcifications and / or multifocal tumors are not eligible for this type of treatment. Chemotherapy is currently the standard treatment and includes the prescription of an anthracycline and a taxane for a duration of 6 to 8 cycles. Dose-dense shemas are of interest in these young patients. The reduction in the interval between the chemotherapies makes it possible to avoid the tumor repopulation between the cycles [12]. Trastuzumab (Herceptin ${ }^{\circledR}$ ) is associated with chemotherapy and continued for one year in the case of HER2 overexpressing tumors [13]. A recent therapeutic option, including docetaxel, carboplatin, trastuzumab (TCH), avoids the use of anthracyclines and thus reduces cardiotoxicity [14]. In case of adverse histologic response after neoadjuvant chemotherapy, no adjuvant chemotherapy is validated. Before any chemotherapy, cryopreservation of oocytes and ovarian follicles is to be proposed in young patients concerned about their fertility. The use of GnRh analogues for the preservation of chemically-induced fertility has not demonstrated its efficacy and oncologic safety, particularly in patients with hormone-dependent tumors, and is only to be proposed in the context of studies [15].

\section{Hormonetherapy}

The hormone therapy currently recommended in young women is tamoxifen for a period of 5 years. The benefit of chemo-induced amenorrhea (stopping the menstrual period $>6$ months after treatment) remains controversial. Ovarian suppression is also discussed. Most teams recommend 2 to 3 years of therapy with LH-RH inhibitor (Zoladex®). In case of contraindication to tamoxifen, treatment with LH-RH inhibitor alone is an alternative.

\section{CONCLUSION}

Breast cancer is a serious disease whose incidence in young women is constantly increasing. This work on breast cancer in young women under 35 years has allowed us to distinguish its different epidemiological, clinical, therapeutic and prognostic characteristics. Young women with breast cancer have a poorer prognosis than their older counterparts. This difference is explained not only by a different distribution of stages at diagnosis, but also by an increased frequency of biological characteristics of tumor aggressiveness. Progress is to be made by multidisciplinary management by experienced teams and participation in research protocols.

Conflicts of Interest: The authors do not declare any conflict of interest.

Authors Contributions: All authors contributed to the critical review of the work. All also read and approved the final version of the manuscript.

\section{REFERENCES}

1. Assi, H. A., Khoury, K. E., Dbouk, H., Khalil, L. E., Mouhieddine, T. H., \& El Saghir, N. S. (2013). Epidemiology and prognosis of breast cancer in young women. Journal of thoracic disease, 5(Suppl 1):2-8.

2. Bouchardy, C., Fioretta, G., Verkooijen, H. M., Vlastos, G., Schaefer, P., Delaloye, J. F., ... \& Chappuis, P. (2007). Recent increase of breast cancer incidence among women under the age of forty. British journal of cancer,96(11), $1743-$ 1746.

3. Molinié, F., Daubisse-Marliac, L., Delafosse, P., \& Tretarre, B. (2010). Épidémiologie du cancer du sein de la femme jeune. $32^{\circ}$ Journées de la Société Française de Sénologie et de Pathologie Mammaire. Journées, Strasbourg, FRA, 2010-1103: La femme jeune face au cancer du sein (Strasbourg, 3-5 novembre 2010)/Young woman facing breast cancer.

4. Cancello, G., Maisonneuve, P., Rotmensz, N., Viale, G., Mastropasqua, M. G., Pruneri, G., ... \& Intra, M. (2010). Prognosis and adjuvant treatment effects in selected breast cancer subtypes of very young women $(<35$ years) with operable breast cancer. Annals of oncology, 21(10), 1974-1981.

5. Silver, D. P., Richardson, A. L., Eklund, A. C., Wang, Z. C., Szallasi, Z., Li, Q., ... \& Fatima, A. (2010). Efficacy of neoadjuvant Cisplatin in triple- 
negative breast cancer. Journal of clinical oncology, 28(7), 1145-1153.

6. Ashworth, A. (2008). A synthetic lethal therapeutic approach: poly (ADP) ribose polymerase inhibitors for the treatment of cancers deficient in DNA double-strand break repair. Journal of Clinical Oncology,26(22), 3785-3790.

7. Voduc, K. D., Cheang, M. C., Tyldesley, S., Gelmon, K., Nielsen, T. O., \& Kennecke, H. (2010). Breast cancer subtypes and the risk of local and regional relapse. Journal of clinical oncology, 28(10), 1684-1691.

8. Coucke, P., Vavassis, P., Vanderick, J. E. A. N., \& Jerusalem, G. (2009). Tumor Margin after conservative breast cancer surgery for early disease: an issue or not?. Belgian Journal of Medical Oncology, 3(3), 93-100.

9. Chang, H. R. (2010). Trastuzumab-based neoadjuvant therapy in patients with HER2positive breast cancer. Cancer, 116(12), 28562867.

10. Sardanelli, F., Boetes, C., Borisch, B., Decker, T., Federico, M., Gilbert, F. J., ... \& Mansel, R. E. (2010). Magnetic resonance imaging of the breast: recommendations from the EUSOMA working group. European journal of cancer, 46(8), 12961316.
11. Bonilla, L., Ben-Aharon, I., Vidal, L., GafterGvili, A., Leibovici, L., \& Stemmer, S. M. (2010). Dose-dense chemotherapy in nonmetastatic breast cancer: a systematic review and meta-analysis of randomized controlled trials. Journal of the National Cancer Institute, 102(24), 1845-1854.

12. Romond, E. H., Perez, E. A., Bryant, J., Suman, V. J., Geyer Jr, C. E., Davidson, N. E., ... \& Swain, S. M. (2005). Trastuzumab plus adjuvant chemotherapy for operable HER2-positive breast cancer. New England Journal of Medicine, 353(16), 1673-1684..

13. Chang, H. R., Slamon, D., Prati, R., Glaspy, J., Pegram, M. D., Kass, F. C., ... \& Chung, D. (2006). A phase II study of neoadjuvant docetaxel/carboplatin with or without trastuzumab in locally advanced breast cancer: Response and cardiotoxicity. Journal of Clinical Oncology, 24(18_suppl), 10515-10515.

14. Azim Jr, H. A., De Azambuja, E., Colozza, M., Bines, J., \& Piccart, M. J. (2011). Long-term toxic effects of adjuvant chemotherapy in breast cancer. Annals of oncology, 22(9), 1939-1947.

15. Ismail-Khan, R., Minton, S., Cox, C., Sims, I., Lacevic, M., Gross-King, M., ... \& Munster, P. N. (2008). Preservation of ovarian function in young women treated with neoadjuvant chemotherapy for breast cancer: a randomized trial using the $\mathrm{GnRH}$ agonist (triptorelin) during chemotherapy. Journal of Clinical Oncology, 26(15_suppl), 524-524. 